\section{§38. Molecular Dynamics Simulation of Micelle Formation in Amphiphilic Solution}

Fujiwara, S., Hashimoto, M ., Itoh, T. (K yoto Inst. Tech.)

Amphiphilic molecules such as lipid molecules and surfactant molecules contain both a hydrophilic "head" and a hydrophobic "tail". In aqueous or organic solvents, these molecules often spontaneously self-assemble into various structures such as spherical and cylindrical micelles, bilayer membranes and bicontinuous cubic structure ${ }^{1)}$. Self-assembly of amphiphilic molecules plays an important role in many biological and industrial processes. For example, surfactants have been successfully used to render quantum dots biocompatible. Although numerous computer simulation studies have so far been carried out on amphiphilic solutions, little is known about the detailed molecular mechanisms of micelle formation in amphiphilic solution. With a view to investigating micelle formation in amphiphilic solution at the molecular level, we perform the molecular dynamics (MD) simulations of coarse-grained amphiphilic molecules with explicit solvent molecules and analyze the micelle formation process.

The computational model is based on those used in the work by Goetz et al. ${ }^{2)}$ and by Noguchi $e t a l^{3)}$. An amphiphilic molecule consists of one hydrophilic particle and two hydrophobic particles which are fixed on a line and spaced equally. A solvent molecule is modeled as a hydrophilic particle. The interaction between a hydrophilic particle and a hydrophobic particle is modeled by a repulsive soft core potential and all other interactions are modeled by a Lennard-Jones potential. The equations of motion for all particles are solved numerically using the leap-frog algorithm at constant temperature with a time step of $\Delta t^{*}=0.0025$. We apply the periodic boundary conditions. The amphiphilic concentration is set to 0.05 . Initially, we provide a randomly distributed configuration of 97 amphiphilic molecules in solution at high temperature $\left(T^{*}=10\right)$ for various number densities $\left(\rho^{*}=0.6,0.65,0.7,0.75,0.8,0.85\right)$. The number of solvent molecules is 5541. The system is then quenched to $T^{*}=1.3$ and MD simulations of $t^{*}=2.0 \times 10^{4}\left(8.0 \times 10^{6}\right.$ time steps $)$ are carried out for each number density.

We show, in Fig. 1, the snapshots of micelles formed at $\rho^{*}=0.7$ and 0.75 . Our simulations indicate that cylindrical micelles are formed at lower densities $\left(\rho^{*} \leq\right.$ 0.7 ) whereas planar bilayers are obtained at higher densities $\left(\rho^{*} \geq 0.75\right)$. The time evolution of the potential energy at $\rho^{*}=0.7$ and 0.75 is shown in Fig. 2. We find from this figure that the potential energy relaxes in a stepwise manner at $\rho^{*}=0.75$. This kind of stepwise behavior is found at $\rho^{*} \geq 0.75$. It is also found that the energy relaxation proceeds in a gradual manner at $\rho^{*}=0.7$. The similar gradual behavior is found at $\rho^{*} \leq 0.7$. These simulation results indicate that the number density has a major effect not only on the micelle shape but also on the micelle formation process.

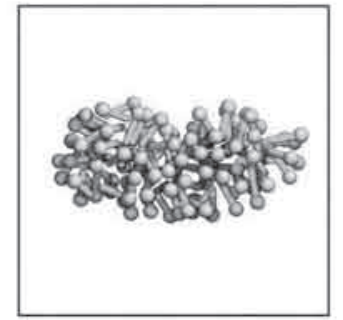

(a)

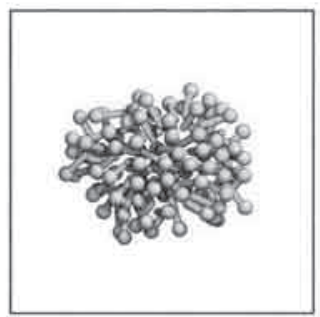

(b)
Fig. 1. Snapshots of micelles formed by amphiphilic molecules in solution: (a) cylindrical micelle $\left(\rho^{*}=0.7\right)$ and (b) planar bilayer $\left(\rho^{*}=0.75\right)$. Hydrophilic and hydrophobic groups are represented by spheres and cylinders, respectively. Solvent molecules are not shown for clarity.
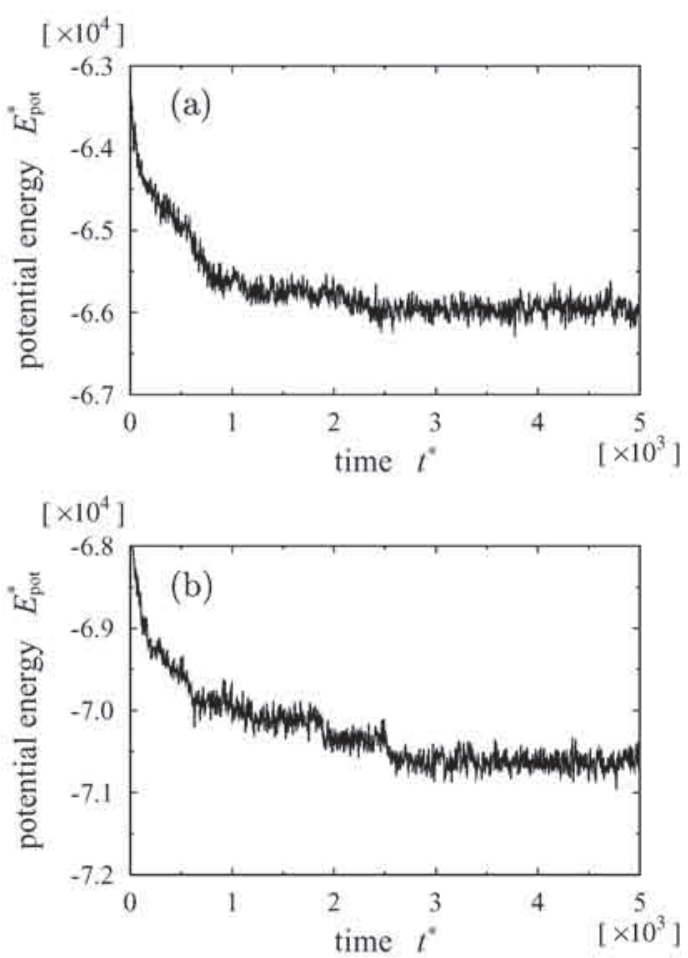

Fig. 2. Time evolution of the potential energy (a) at $\rho^{*}=0.7$ and (b) at $\rho^{*}=0.75$.

\section{References}

1) Structure and Dynamics of Membranes, edited by Lipowsky, R. and Sackmann, E. (Elsevier Science, Amsterdam, 1995).

2) Goetz, R. and Lipowsky, R., J. Chem. Phys. 108, (1998) 7397.

3) Noguchi, H. and Takasu, M., Phys. Rev. E 64, (2001) 041913. 